\title{
Dynamics of warm power-law plateau inflation with a generalized inflaton decay rate: predictions and constraints after Planck 2015
}

\author{
Abdul Jawad ${ }^{1, a}$, Nelson Videla ${ }^{2, b}$, Faiza Gulshan ${ }^{3, c}$ \\ ${ }^{1}$ Department of Mathematics, COMSATS Institute of Information Technology, Lahore 54000, Pakistan \\ ${ }^{2}$ Departamento de Física, FCFM, Universidad de Chile, Blanco Encalada 2008, Santiago, Chile \\ ${ }^{3}$ Department of Mathematics, Lahore Leads University, Lahore 54000, Pakistan
}

Received: 20 January 2017 / Accepted: 21 April 2017 / Published online: 29 April 2017

(C) The Author(s) 2017. This article is an open access publication

\begin{abstract}
In the present work, we study the consequences of considering a new family of single-field inflation models, called power-law plateau inflation, in the warm inflation framework. We consider the inflationary expansion is driven by a standard scalar field with a decay ratio $\Gamma$ having a generic power-law dependence with the scalar field $\phi$ and the temperature of the thermal bath $T$ given by $\Gamma(\phi, T)=C_{\phi} \frac{T^{a}}{\phi^{a-1}}$. Assuming that our model evolves according to the strong dissipative regime, we study the background and perturbative dynamics, obtaining the most relevant inflationary observable as the scalar power spectrum, the scalar spectral index and its running and the tensor-toscalar ratio. The free parameters characterizing our model are constrained by considering the essential condition for warm inflation, the conditions for the model evolves according to the strong dissipative regime and the 2015 Planck results through the $n_{\mathrm{s}}-r$ plane. For completeness, we study the predictions in the $n_{\mathrm{s}}-\mathrm{d} n_{\mathrm{s}} / \mathrm{d} \ln k$ plane. The model is consistent with a strong dissipative dynamics and predicts values for the tensor-to-scalar ratio and for the running of the scalar spectral index consistent with current bounds imposed by Planck and we conclude that the model is viable.
\end{abstract}

\section{Introduction}

The inflationary universe has become in the most acceptable framework in describing the physics of the very early universe. Besides of solving most of the shortcomings of the hot big-bang scenario, like the horizon, the flatness and the monopole problems [1-7], inflation also gener-

\footnotetext{
a e-mail: abduljawad@ciitlahore.edu.pk; jawadab181@yahoo.com

b e-mail: nelson.videla@ing.uchile.cl

c e-mail: fazi.gull@yahoo.com
}

ates a mechanism to explain the large-scale structure (LSS) of the universe [8-13] and the origin of the anisotropies observed in the cosmic microwave background (CMB) radiation [14-21], since primordial density perturbations may be sourced from quantum fluctuations of the inflaton scalar field during the inflationary expansion. The standard cold inflation scenario is divided into two regimes: the slowroll and reheating phases. In the slow-roll period the universe undergoes an accelerated expansion and all interactions of the inflaton scalar field with other field degrees of freedom are typically neglected. Subsequently, a reheating period [22-25] is invoked to end the brief acceleration. After reheating, the universe is filled with relativistic particles and thus the universe enters in the radiation big-bang epoch.

Upon comparison to the current cosmological and astronomical observations, specially those related with the $\mathrm{CMB}$ temperature anisotropies, it is possible to constrain the inflationary models. In particular, the constraints in the $n_{\mathrm{s}}-r$ plane give us the predictions of a number of representative inflationary potentials. Recently, the Planck collaboration has published new data of enhanced precision of the CMB anisotropies [21]. Here, the Planck full mission data has improved the upper bound on the tensor-to-scalar ratio $r_{0.002}<0.11(95 \% \mathrm{CL})$ which is similar to obtained from [19], in which $r<0.12$ (95\% CL). In particular, some representative models, as chaotic inflation, which predict a large value of the tensor-to-scalar ratio $r$, are ruled out by the data. As reported in Ref. [26], the Planck data tends to support plateau-like inflaton scalar potentials, which are asymptotically constant. Starobinsky $R^{2}$ [1] and Higgs inflation [27] are the most representative models with this class of potentials and, more recently, the $\alpha$-attractors models $[28,29]$. For this last class of models, the approach to the inflationary plateau is exponential, being indistinguishable from Starobinsky and Higgs inflation models. In this direc- 
tion, starting from global supersymmetry and considering a superpotential, there was proposed in [30,31] a new class of models called shaft inflation. As opposed to Starobinsky and Higgs inflation, the approach to the plateau is of powerlaw type. In a subsequent work [32], another new family of inflationary models is studied, proposing a phenomenological potential

$V(\phi)=V_{0}\left[\frac{\left(\frac{\phi}{M_{\mathrm{p}}}\right)^{n}}{\left(\frac{\phi}{M_{\mathrm{p}}}\right)^{n}+\alpha^{n}}\right]^{q}$,

where $n$ and $q$ are real parameters, $V_{0}$ is a constant density scale and $\alpha \equiv \frac{M}{M_{\mathrm{p}}}$, with $M$ being a mass scale and $M_{\mathrm{p}}=2.43 \times 10^{18} \mathrm{GeV}$ denotes the reduced Planck mass. In addition, it is required that $\phi \geq M$ or equivalently $\phi \geq \alpha M_{\mathrm{p}}$; otherwise this model is indistinguishable from monomial inflation where $V \propto \phi^{n q}$. It was demonstrated in the $r-n_{\mathrm{s}}$ plane that the predictions of power-law plateau inflation are distinct and testable compared to several inflation models. In particular, the case $n=2$ and $q=1$ corresponds to the best model choice in Ref. [32]. Despite this attractiveness, in order to be a realistic model, it needs to be embedded in a convenient theoretical framework.

On the other hand, some classes of inflaton models excluded by current data in the standard cold inflation scenario which can be saved in the warm inflation scenario, which is an alternative mechanism for having successful inflation. The warm inflation scenario, as opposed to standard cold inflation, has the essential feature that a reheating phase is avoided at the end of the accelerated expansion due to the decay of the inflaton into radiation and particles during the slow-roll phase [33-35]. During warm inflation, the temperature of the universe does not drop dramatically and the universe can smoothly enter into the decelerated, radiation-dominated period, which is essential for a successful big-bang nucleosynthesis. In the warm inflation scenario, dissipative effects are important during the accelerated expansion, so that radiation production occurs concurrently with the accelerated expansion. For a representative list of recent references see Refs. [36-45]. The dissipative effect arises from a friction term or dissipative coefficient $\Gamma$, which describes the processes of the scalar field dissipating into a thermal bath via its interaction with other field degrees of freedom. The effectiveness of warm inflation may be parametrized by the ratio $R \equiv \Gamma / 3 H$. The weak dissipative regime for warm inflation is for $R \ll 1$ while for $R \gg 1$, it is the strong dissipative regime for warm inflation. It is important to emphasize that the dissipative coefficient $\Gamma$ may be computed from first principles in quantum field theory considering that $\Gamma$ encodes the microscopic physics resulting from the interactions between the inflaton and the other fields that can be present. For instance, by considering different decay mechanisms, it is possible to obtain several expressions for the dissipative coefficient $\Gamma$. In particular, in Refs. [46-48], a supersymmetric model containing three superfields $\Phi, X$, and $Y$ has been studied, with a superpotential $W=f(\Phi)+\frac{g}{2} \Phi X^{2}+\frac{h}{2} X Y^{2}$, where the scalar components of the superfields are $\phi=\sqrt{2}\langle\Phi\rangle, \chi$, and $y$ respectively. The inflaton scalar potential is given by $V(\phi)=\left|f^{\prime}(\phi)^{2}\right|$, which spontaneously breaks supersymmetry (SUSY). By coupling the inflaton to the bosonic and fermionic $X$ fields and their subsequent decay into $Y$ scalars and fermions, which form the thermal bath, and for the case of low-temperature regime, when the mass of the catalyst field $m_{\chi}$ is larger than the temperature $T$, the resulting dissipation coefficient can be well described by the expression $\Gamma=C_{\phi} \frac{T^{3}}{\phi^{2}}$, where $C_{\phi}$ is a dimensionless parameter related to the dissipative microscopic dynamics. For this particular case $C_{\phi} \simeq \frac{1}{4} \alpha_{h} N_{X}$, with $\alpha_{\mathrm{h}}=h^{2} N_{Y} / 4 \pi \lesssim 1$ and $N_{X, Y}$ denote the multiplicity of chiral superfields. In this direction, SUSY ensures that quantum and thermal corrections to the effective potential are under control $[49,50]$. As another example, in Ref. [51], it was demonstrated that a form $\Gamma=C_{\phi} \phi^{2} / T$, in principle, with problems of large thermal corrections for the models studied in Refs. [52,53], may produce a consistent warm inflation scenario for a specific model. On the other hand, it was shown for the first time in Ref. [39] that warm inflation can be realized by directly coupling the inflaton to a few light fields, instead of considering indirect couplings to light fields through heavy mediator fields, as in Refs. [46-48]. Then the expression obtained for $\Gamma$ turns out to be $\Gamma=C_{\phi} T$.

Following Refs. [47,54,55], a general parametrization of the dissipative coefficient $\Gamma(T, \phi)$ can be written as

$\Gamma(\phi, T)=C_{\phi} \frac{T^{a}}{\phi^{a-1}}$

This expression for $\Gamma$ includes the several cases mentioned above. Specifically, for the value $a=3$, this case corresponds to a low temperature regime, when the mass of the catalyst field $m_{\chi}$ is larger than the temperature $T$ [46-48]. On the other hand, $a=1$, i.e., $\Gamma \propto T$ corresponds [39]. For $a=0$, the dissipative coefficient represents an exponentially decaying propagator in the high temperature regime. Finally, for $a=-1, \Gamma \propto \phi^{2} / T$ agrees with the non-SUSY case [51-53].

Additionally, thermal fluctuations during the inflationary scenario may play a fundamental role in producing the primordial fluctuations [56-58]. In the warm inflationary scenario the density perturbations arise from thermal fluctuations of the inflaton and dominate over the quantum ones. In this form, an essential condition for warm inflation to occur is the existence of a radiation component with temperature 
$T>H$, since the thermal and quantum fluctuations are proportional to $T$ and $H$, respectively [33-35,56-62]. When the universe heats up and becomes radiation dominated, inflation ends and the universe smoothly enters in the radiation bigbang phase $[33,34]$. For a comprehensive review of warm inflation; see Refs. [49,50]. In this direction, there are many phenomenological models of warm inflation, but more interesting are the first principles models of warm inflation in which the dissipative coefficient and effective potential are computed from quantum field theory. For instance, in Refs. $[49,50]$, the following inflaton superpotential $W(\Phi)$ was considered:

$W(\Phi)=\frac{\lambda}{p+1} \frac{\Phi^{p+1}}{m_{\mathrm{p}}^{p-2}}$,

which reduces to chaotic inflation models for $p>1$. In particular, in Ref. [48] the authors studied the quartic potential $V(\phi)=\lambda \phi^{4}$, which corresponds to a superpotential $W(\phi)=\lambda \Phi^{3} / 3$ together with the dissipative coefficient corresponding to $a=3$, i.e. $\Gamma \propto T^{3} / \phi^{2}$, was confronted with current data available at that time. On the other hand, when $p=0$ and $\lambda<0$, we have the standard supersymmetric hybrid inflation.

Given the attractiveness of the power-law plateau inflation models as a new class of candidates in describing inflation, the main goal of this work is study the consequences of considering this new family of single-field inflation models in the warm inflation scenario in order to avoid the reheating phase. We would like to emphasize that our analysis is phenomenological in the sense that, in order to describe the dissipative effects during the inflationary expansion, we consider the generalized parametrization for the inflaton decay rate given by Eq. (2) and without considering a first principles construction for our model. However, in Ref. [32], the authors presented a toy-model in supergravity (SUGRA) which can produce the scalar potential of Plateau inflation (1) for $n=2$ and $q=1$. Specifically, they considered only global supersymmetry (SUSY) and sub-Planckian fields with the superpotential

$W=\frac{S^{2}\left(\Phi_{1}^{2}-\Phi_{2}^{2}\right)}{2 m}$,

where $S, \Phi_{1}, \Phi_{2}$ are chiral superfields and $m$ is a large, but sub-Planckian scale. An interesting approach could be specify a decaying mechanism for the scalar components of the superfields $\Phi_{1}$ and $\Phi_{2}$ in light fields which form the thermal bath and compute the corresponding dissipative coefficient $\Gamma$ starting form first principles. However, this further considerations go beyond the scope of our present work, but these may be regarded as basis of a future work. On the other hand, we will restrict ourselves only to the study of the strong dis- sipative regime, $R \gg 1$. For this dissipative regime, in the slow-roll approximation, we study the background as well as the perturbative dynamics. The free parameters characterizing our model are constrained by considering the essential condition for warm inflation, $T>H$, the condition for the model evolves according to strong dissipative regime and the 2015 Planck results through the $n_{\mathrm{s}}-r$ plane. For completeness, we study the predictions of our model regarding the running of the scalar spectral index through the $n_{\mathrm{s}}-\mathrm{d} n_{\mathrm{s}} / \mathrm{d} \ln k$ plane.

This paper is organized as follows: In the next section, we present the basic setup of warm inflation. In Sect. 3, we study the background and perturbative dynamics when our model evolves according to strong regime. Specifically, we find explicit expressions for the most relevant inflationary observable as the scalar power spectrum, scalar spectral index, the running of the scalar spectral index and the tensor-to-scalar ratio. In order to establish a direct comparison between the power-law plateau inflation in the cold and warm scenarios, we will restrict ourselves to the case $n=2$ and $q=1$. For this particular case, we obtain the predictions in the $n_{\mathrm{s}}-r$ and $n_{\mathrm{s}}-\mathrm{d} n_{\mathrm{s}} / \mathrm{d} \ln k$ plane.

Finally, Sect. 4 summarizes our finding and exhibits our conclusions. We have chosen units such that $c=\hbar=1$.

\section{Basics of warm inflation scenario}

In this section, we introduce the basic setup of warm inflation

\subsection{Background evolution}

We start by considering a spatially flat FriedmannRobertson-Walker (FRW) universe containing a self-interacting inflaton scalar field $\phi$ with energy density and pressure given by $\rho_{\phi}=\dot{\phi}^{2} / 2+V(\phi)$ and $P_{\phi}=\dot{\phi}^{2} / 2-V(\phi)$, respectively and a radiation field with energy density $\rho_{\gamma}$. The corresponding Friedmann equation reads

$$
H^{2}=\frac{1}{3 M_{\mathrm{p}}^{2}}\left(\rho_{\phi}+\rho_{\gamma}\right)
$$

where $M_{\mathrm{p}}=\frac{1}{\sqrt{8 \pi G}}$ is the reduced Planck mass.

The dynamics of $\rho_{\phi}$ and $\rho_{\gamma}$ is described by the equations [33-35]

$\dot{\rho_{\phi}}+3 H\left(\rho_{\phi}+P_{\phi}\right)=-\Gamma \dot{\phi}^{2}$,

and

$\dot{\rho}_{\gamma}+4 H \rho_{\gamma}=\Gamma \dot{\phi}^{2}$, 
where the dissipative coefficient $\Gamma>0$ produces the decay of the scalar field into radiation. Recall that this decay rate can be assumed to be a function of the temperature of the thermal bath $\Gamma(T)$, or a function of the scalar field $\Gamma(\phi)$, or a function of $\Gamma(T, \phi)$ or simply a constant. As we have mentioned in the introduction, the parametrization given by Eq. (2) includes different cases, depending of the values of $a$. Particularly, the inflaton decay rates $a=3\left(\Gamma=C_{\phi} \frac{T^{3}}{\phi^{2}}\right)$ and $a=1\left(\Gamma=C_{\phi} T\right)$ have been studied extensively in the

literature $[38,43,47,65-69]$.

During warm inflation, the energy density related to the scalar field predominates over the energy density of the radiation field, i.e., $\rho_{\phi} \gg \rho_{\gamma}$ [33-35,56-61], but even if small when compared to the inflaton energy density it can be larger than the expansion rate with $\rho_{\gamma}^{1 / 4}>H$. Assuming thermalization, this translates roughly into $T>H$, which is the condition for warm inflation to occur.

When $H, \phi$, and $\Gamma$ are slowly varying, which is a good approximation during inflation, the production of radiation becomes quasi-stable, i.e., $\dot{\rho}_{\gamma} \ll 4 H \rho_{\gamma}$ and $\dot{\rho}_{\gamma} \ll \Gamma \dot{\phi}^{2}$; see Refs. [33-35,56-61]. Then the equations of motion reduce to

$$
3 H(1+R) \dot{\phi} \simeq-V_{, \phi},
$$

where, $\phi$ denotes differentiation with respect to inflaton, and

$4 H \rho_{\gamma} \simeq \Gamma \dot{\phi}^{2}$,

where $R$ is the dissipative ratio defined as

$R \equiv \frac{\Gamma}{3 H}$

In warm inflation, we can distinguish between two possible scenarios, namely the weak and strong dissipative regimes, defined as $R \ll 1$ and $R \gg 1$, respectively. In the weak dissipative regime, the Hubble damping is still the dominant term, however, in the strong dissipative regime, the dissipative coefficient $\Gamma$ controls the damped evolution of the inflaton field.

If we consider thermalization, then the energy density of the radiation field could be written as $\rho_{\gamma}=C_{\gamma} T^{4}$, where the constant $C_{\gamma}=\pi^{2} g_{*} / 30$. Here, $g_{*}$ represents the number of relativistic degrees of freedom. In the Minimal Supersymmetric standard model (MSSM), $g=228.75$ and $C_{\gamma} \simeq 70$ [60]. Combining Eqs. (8) and (9) with $\rho_{\gamma} \propto T^{4}$, the temperature of the thermal bath becomes

$T=\left[\frac{\Gamma V_{, \phi}^{2}}{36 C_{\gamma} H^{3}(1+R)^{2}}\right]^{1 / 4}$.

On the other hand, the consistency conditions for the approximations to hold imply that a set of slow-roll con- ditions must be satisfied for a prolonged period of inflation to take place. For warm inflation, the slow-roll parameters are $[46,60]$

$\epsilon=\frac{M_{\mathrm{p}}^{2}}{2}\left(\frac{V_{, \phi}}{V}\right)^{2}, \quad \eta=M_{\mathrm{p}}^{2}\left(\frac{V_{, \phi \phi}}{V}\right)$,

$\beta=M_{\mathrm{p}}^{2}\left(\frac{\Gamma_{, \phi} V_{, \phi}}{\Gamma V}\right), \quad \sigma=M_{\mathrm{p}}^{2}\left(\frac{V_{, \phi}}{\phi V}\right)$.

The slow-roll conditions for warm inflation can be expressed as $[46,60,61]$

$\epsilon \ll 1+R, \quad \eta \ll 1+R, \quad \beta \ll 1+R, \quad \sigma \ll 1+R$

When one these conditions is not longer satisfied, either the motion of the inflaton is no longer overdamped and slowroll ends, or the radiation becomes comparable to the inflaton energy density. In this way, inflation ends when one of these parameters become the order of $1+R$.

The number of $e$-folds in the slow-roll approximation, using (5) and (8), yields

$N \simeq-\frac{1}{M_{\mathrm{p}}^{2}} \int_{\phi_{*}}^{\phi_{\text {end }}} \frac{V}{V_{, \phi}}(1+R) \mathrm{d} \phi$,

where $\phi_{*}$ and $\phi_{\text {end }}$ are the values of the scalar field when the cosmological scales crosses the Hubble-radius and at the end of inflation, respectively. As it can be seen, the number of $e$-folds is increased due to an extra term of $(1+R)$. This implies a more amount of inflation, between these two values of the field, compared to cold inflation.

\subsection{Cosmological perturbations}

In the warm inflation scenario, a thermalized radiation component is present with $T>H$, then the inflaton fluctuations $\delta \phi$ are predominantly thermal instead quantum. In this way, following $[49,50,57,60,61]$, the amplitude of the power spectrum of the curvature perturbation is given by

$\mathcal{P}_{\mathcal{R}}{ }^{1 / 2} \simeq\left(\frac{H}{2 \pi}\right)\left(\frac{3 H^{2}}{V_{, \phi}}\right)(1+R)^{5 / 4}\left(\frac{T}{H}\right)^{1 / 2}$,

where the normalization has been chosen in order to recover the standard cold inflation result when $R \rightarrow 0$ and $T \simeq H$.

By the other hand, the scalar spectral index $n_{\mathrm{s}}$ to leading order in the slow-roll approximation is given by $[60,61]$

$$
\begin{aligned}
n_{\mathrm{s}} & =1+\frac{\mathrm{d} \ln \mathcal{P}_{\mathcal{R}}}{\mathrm{d} \ln k} \\
& \simeq 1-\frac{(17+9 R)}{4(1+R)^{2}} \epsilon-\frac{(1+9 R)}{4(1+R)^{2}} \beta+\frac{3}{2(1+R)} \eta
\end{aligned}
$$


We also introduce the running of the scalar spectral index, which represents the scale dependence of the spectral index, by $n_{\text {run }}=\frac{\mathrm{d} n_{\mathrm{s}}}{\mathrm{d} \ln k}$. In particular, for the strong dissipative regime, this expressions is given by $[60,61]$

$$
\begin{gathered}
\frac{\mathrm{d} n_{\mathrm{s}}}{\mathrm{d} \ln k} \simeq \frac{1}{R^{2}}\left(-\frac{9}{2} \beta^{2}-\frac{27}{4} \epsilon^{2}-\frac{9}{2} \epsilon \beta+\frac{15}{4} \eta \beta\right. \\
\left.+6 \epsilon \eta-\frac{3}{2} \zeta^{2}+\frac{9}{2} \gamma \epsilon\right),
\end{gathered}
$$

where $\zeta^{2}$ and $\gamma$ are second-order slow-roll parameters defined by

$\zeta^{2} \equiv M_{\mathrm{p}}^{4}\left(\frac{V_{, \phi} V_{, \phi \phi \phi}}{V^{2}}\right)$

and

$\gamma \equiv M_{\mathrm{p}}^{2}\left(\frac{\Gamma_{, \phi \phi}}{\Gamma}\right)$

respectively.

Regarding to tensor perturbations, these do not couple to the thermal background, so gravitational waves are only generated by quantum fluctuations, as in standard inflation $[62,63]$. However, the tensor-to-scalar ratio $r$ is modified with respect to standard cold inflation, yielding $[49,50]$

$r \simeq\left(\frac{H}{T}\right) \frac{16 \epsilon}{(1+R)^{5 / 2}}$.

We can see that warm inflation predicts a tensor-to-scalar ratio suppressed by a factor $(T / H)(1+R)^{5 / 2}>1$ compared with standard cold inflation.

When a specific form of the scalar potential and the dissipative coefficient $\Gamma$ are considered, it is possible to study the background evolution under the slow-roll regime and the primordial perturbations in order to test the viability of warm inflation. In the following we will study how an inflaton decay rate with a generic power-law dependence with the scalar field $\phi$ and the temperature of the thermal bath $T$ influences the inflationary dynamics for the power-law plateau potential. We will restrict ourselves to the strong dissipation regime.

\section{Dynamics of warm power-law plateau inflation in the strong dissipative regime}

\subsection{Background evolution}

Assuming that the inflationary dynamics takes place in the strong dissipative regime, i.e., $R \gg 1$ ( or $\Gamma \gg 3 H$ ), by using Eqs. (2) and (11), the temperature of the thermal bath as a function of the inflaton field is found to be

$$
\begin{aligned}
T= & {\left[4 \sqrt{3} C_{\phi}^{-1}\left(n q \alpha^{n}\right)^{2}\right]^{\frac{1}{4+a}}\left(V_{0}^{\frac{3}{2}} M_{\mathrm{p}}^{a-2}\right)^{\frac{1}{4+a}} } \\
& \times\left(\frac{\phi}{M_{\mathrm{p}}}\right)^{\frac{3(n q-2)+2 a}{2(4+a)}}\left(\alpha^{n}+\left(\frac{\phi}{M_{\mathrm{p}}}\right)^{n}\right)^{-\frac{(4+3 q)}{2(4+a)}} .
\end{aligned}
$$

Replacing the last expression into Eq. (2), both the inflaton decay rate and the ratio $R=\Gamma / 3 H$ expressed in terms of the inflaton field becomes

$$
\begin{aligned}
\Gamma= & {\left[\left(\sqrt{3}\left(4 C_{\gamma}\right)^{-1}\left(n q \alpha^{n}\right)^{2}\right)^{a} C_{\phi}^{4}\right]^{\frac{1}{4+a}}\left(V_{0}^{\frac{3 a}{2}} M_{\mathrm{p}}^{4-5 a}\right)^{\frac{1}{4+a}} } \\
& \times\left(\frac{\phi}{M_{\mathrm{p}}}\right)^{\frac{8+3 a(n q-4)}{2(4+a)}}\left(\alpha^{n}+\left(\frac{\phi}{M_{\mathrm{p}}}\right)^{n}\right)^{-\frac{a(4+3 q)}{2(4+a)}}
\end{aligned}
$$

and

$$
\begin{aligned}
R= & {\left[\left(\sqrt{3}\left(4 C_{\gamma}\right)^{-1}\left(n q \alpha^{n}\right)^{2}\right)^{a} C_{\phi}^{4}\right]^{\frac{1}{4+a}}\left(\frac{M_{\mathrm{p}}^{4}}{V_{0}}\right)^{\frac{2-a}{4+a}} } \\
& \times\left(\frac{\phi}{M_{\mathrm{p}}}\right)^{\frac{8+3 a(n q-4)}{2(4+a)}-\frac{n q}{2}}\left(\alpha^{n}+\left(\frac{\phi}{M_{\mathrm{p}}}\right)^{n}\right)^{-\frac{a(4+3 q)}{2(4+a)}+\frac{n q}{2}},
\end{aligned}
$$

respectively.

In this way, by combining Eqs. (1), (8), and (22), the inflaton field as a function of cosmic time may be obtained from the following expression:

$$
\begin{aligned}
& {\left[\sqrt{3}\left(4 C_{\gamma}\right)^{-a}\left(n \alpha^{n}\right)^{-8} C_{\phi}^{4} q^{a-4}\right]^{\frac{1}{4+a}} g_{0}^{-1}} \\
& \quad \times\left(V_{0}^{-\frac{1}{2}-\frac{6}{4+a}} M_{\mathrm{p}}^{\frac{3(4-a)}{4+a}}\right)\left(\frac{\phi}{M_{\mathrm{p}}}\right)^{n g_{0}}\left(\alpha^{n}+\left(\frac{\phi}{M_{\mathrm{p}}}\right)^{n}\right)^{\frac{q(8-a)+16}{2(4+a)}} \\
& \quad \times{ }_{2} F_{1}\left[1, \frac{12-4 a+8 n}{n(4+a)}, 1+g_{0},-\alpha^{-n}\left(\phi / M_{\mathrm{p}}\right)^{n}\right]=-t,
\end{aligned}
$$

where $g_{0} \equiv \frac{24-8 n q-8 a+a n q}{2 n(4+a)}$ and ${ }_{2} F_{1}$ denotes the hypergeometric function [64].

For this model the set of slow-roll parameters become

$$
\begin{aligned}
\epsilon= & \frac{\left(n q \alpha^{n}\right)^{2}}{2\left(\frac{\phi}{M_{\mathrm{p}}}\right)^{2}\left(\alpha^{n}+\left(\frac{\phi}{M_{\mathrm{p}}}\right)^{n}\right)^{2}}, \\
\eta= & \frac{n q \alpha^{n}}{\left(\frac{\phi}{M_{\mathrm{p}}}\right)^{2} \frac{\left[(n q-1) \alpha^{n}-(n+1)\left(\frac{\phi}{M_{\mathrm{p}}}\right)^{n}\right]}{\left(\alpha^{n}+\left(\frac{\phi}{M_{\mathrm{p}}}\right)^{n}\right)^{2}}} \\
\beta= & \frac{n q \alpha^{n}}{2(4+a)\left(\frac{\phi}{M_{\mathrm{p}}}\right)^{2}} \\
& \times \frac{\left[(8+3 a(n q-4)) \alpha^{n}-4(a(3+n)-2)\left(\frac{\phi}{M_{\mathrm{p}}}\right)^{n}\right]}{\left(\alpha^{n}+\left(\frac{\phi}{M_{\mathrm{p}}}\right)^{n}\right)^{2}},
\end{aligned}
$$


$\sigma=\frac{n q \alpha^{n}}{\left(\frac{\phi}{M_{\mathrm{p}}}\right)^{2}\left(\alpha^{n}+\left(\frac{\phi}{M_{\mathrm{p}}}\right)^{n}\right)}$.

For the strong dissipative regime, the slow-roll conditions (13) become

$\epsilon \ll R, \quad \eta \ll R, \beta \ll R, \sigma \ll R$.

As we mentioned in the previous section, inflation ends when one of these parameters become the order of $R$.

On the other hand, the number of inflationary $e$-folds between the values of the scalar field when a given perturbation scale leaves the Hubble-radius and at the end of inflation can be computed from Eqs. (1) and (23) into (14), yielding

$$
\begin{aligned}
N= & g_{1}^{-1}\left[\left(9\left(4 C_{\gamma}\right)^{a}\left(n \alpha^{n}\right)^{8}\right)^{-1} C_{\phi}^{4} q^{a-4}\right]^{\frac{1}{4+a}} \\
& \times\left(\frac{M_{\mathrm{p}}^{4}}{V_{0}}\right)^{\frac{2-a}{4+a}}\left(\frac{\phi}{M_{\mathrm{p}}}\right)^{n g_{1}}\left(\alpha^{n}+\left(\frac{\phi}{M_{\mathrm{p}}}\right)^{n}\right)^{\frac{8+q(2-a)}{4+a}} \\
& \times\left.{ }_{2} F_{1}\left[1, \frac{12-4 a+8 n}{n(4+a)}, 1+g_{1},-\alpha^{-n}\left(\phi / M_{\mathrm{p}}\right)^{n}\right]\right|_{\phi_{\mathrm{end}}} ^{\phi_{*}},
\end{aligned}
$$

where $g_{1} \equiv \frac{n q(a-2)-4(a-3)}{n(4+a)}$.

Since Eq. (30) has a complicated dependence in the inflaton field, it is not possible to express $\phi_{*}$ as a function of $N$ analytically. Instead, for numerical purposes, from Eq. (1) we may express the inflaton field as a function of the amplitude of the potential and evaluate this expression at the Hubbleradius crossing, obtaining

$\frac{\phi_{*}}{M_{\mathrm{p}}}=\left[\frac{\alpha^{n}\left(\frac{V_{*}}{V_{0}}\right)^{\frac{1}{q}}}{1-\left(\frac{V_{*}}{V_{0}}\right)^{\frac{1}{q}}}\right]^{\frac{1}{n}}$.

The last expression will be useful to evaluate the several inflationary observables and put the observational bounds on our model.

\subsection{Cosmological perturbations}

Now, we shall study the cosmological perturbations for our model in the strong dissipative regime $R=\Gamma / 3 H>1$. For this regime, the amplitude of the scalar power spectrum (32) becomes

$\mathcal{P}_{\mathcal{R}}^{1 / 2} \simeq\left(\frac{H}{2 \pi}\right)\left(\frac{3 H^{2}}{V_{, \phi}}\right)\left(\frac{T}{H}\right)^{1 / 2} R^{5 / 4}$,

then, by replacing Eqs. (1), (21), and (23), the power spectrum as a function of the inflaton field is found to be

$$
\begin{aligned}
\mathcal{P}_{\mathcal{R}}= & \frac{\left(2^{7} C_{\gamma}^{5 / 2}\right)^{-1}}{\pi^{2}}\left[2^{18} \sqrt{3}^{-(13+a)}\left(C_{\gamma} C_{\phi}\right)^{9}\left(n q \alpha^{n}\right)^{3(a-2)}\right]^{\frac{1}{4+a}} \\
& \times\left(\frac{M_{\mathrm{p}}^{4}}{V_{0}}\right)^{\frac{3(1-2 a)}{2(4+a)}}\left(\frac{\phi}{M_{\mathrm{p}}}\right)^{\frac{6(5-a)-3 n q(1-2 a)}{2(4+a)}} \\
& \times\left(\alpha^{n}+\left(\frac{\phi}{M_{\mathrm{p}}}\right)^{n}\right)^{\frac{3[2(2-a)+q(1-2 a)]}{2(4+a)}}
\end{aligned}
$$

By considering the strong dissipative regime, the expression for the scalar spectral index becomes

$n_{\mathrm{s}} \simeq 1+\frac{1}{R}(-9 \epsilon-9 \beta+6 \eta)$,

in this way, by replacing Eqs. (23) and (25)-(27), the inflaton field dependence of the scalar spectral index is given by

$$
\begin{aligned}
n_{\mathrm{S}}= & \frac{1}{4+a}\left[2^{a-4} 3^{6+a} C_{\gamma}^{a} C_{\phi}^{-4}\left(n q \alpha^{n}\right)^{4-a}\right]^{\frac{1}{4+a}} \\
& \times\left(\frac{\phi}{M_{\mathrm{p}}}\right)^{\frac{4(a-3)-n q(a-2)}{4+a}}\left(\alpha^{n}+\left(\frac{\phi}{M_{\mathrm{p}}}\right)^{n}\right)^{\frac{-8+q(a-2)}{4+a}} \\
& \times\left(\frac{M_{\mathrm{p}}^{4}}{V_{0}}\right)^{\frac{a-2}{4+a}}\left[(2(4 a-5)+n q(1-2 a)) \alpha^{n}\right. \\
& \left.+2(4 a-5+n(a-2))\left(\frac{\phi}{M_{\mathrm{p}}}\right)^{n}\right]+1 .
\end{aligned}
$$

Regarding the running of the scalar spectral index, the second-order slow-roll parameters $\zeta^{2}$ and $\gamma$ for this model become

$$
\begin{aligned}
\zeta^{2}= & \frac{\left(n q \alpha^{n}\right)^{2}}{\left(\frac{\phi}{M_{\mathrm{p}}}\right)^{4}\left(\alpha^{n}+\left(\frac{\phi}{M_{\mathrm{p}}}\right)^{n}\right)^{4}} \\
& \times\left[\left(2-3 n q+n^{2} q^{2}\right) \alpha^{2 n}-(1+n)(n-4+3 n q) \alpha^{n}\right. \\
& \left.\times\left(\frac{\phi}{M_{\mathrm{p}}}\right)+\left(2+3 n+n^{2}\right)\left(\frac{\phi}{M_{\mathrm{p}}}\right)^{2 n}\right]
\end{aligned}
$$

and

$$
\begin{aligned}
\gamma= & \frac{a}{4(4+a)^{2}\left(\frac{\phi}{M_{\mathrm{p}}}\right)^{2}\left(\alpha^{n}+\left(\frac{\phi}{M_{\mathrm{p}}}\right)^{n}\right)^{2}} \\
& \times\left[(3 n q-14)(8+3 a(n q-4)) \alpha^{2 n}\right. \\
& -2\left(a\left(-168+13 n(3 q-4)+n^{2}(4+15 q)\right)\right) \\
& \times \alpha^{n}\left(\frac{\phi}{M_{\mathrm{p}}}\right)^{n}+4\left(28+n(4-3 q)+n^{2}(4+3 q)\right) \\
& \left.+8(7+2 n)(a(n+3)-2)\left(\frac{\phi}{M_{\mathrm{p}}}\right)^{2 n}\right],
\end{aligned}
$$


respectively. Then, by replacing last expressions together with Eqs. (25)-(27) into (17), the running of the spectral index is completely determined (not shown).

Regarding the tensor perturbations, the tensor-to-scalar ratio for the strong regime becomes

$r \simeq\left(\frac{H}{T}\right) \frac{16 \epsilon}{R^{5 / 2}}$

The inflaton field dependence of the tensor-to-scalar ratio is determined by replacing Eqs. (1), (11), and (25) into (38), yielding

$$
\begin{aligned}
r= & 2^{8} C_{\gamma}^{5 / 2}\left[2^{-18} \sqrt{3}^{5-a}\left(C_{\gamma} C_{\phi}\right)^{-9}\left(n q \alpha^{n}\right)^{3 n(2-a)}\right]^{\frac{1}{4+a}} \\
& \times\left(\frac{M_{\mathrm{p}}^{4}}{V_{0}}\right)^{\frac{4 a-11}{2(4+a)}}\left(\frac{\phi}{M_{\mathrm{p}}}\right)^{\frac{-30+11 n q-4 a(n q-6)}{2(4+a)}} \\
& \times\left(\alpha^{n}+\left(\frac{\phi}{M_{\mathrm{p}}}\right)^{n}\right)^{\frac{6(a-2)+q(4 a-11)}{2(4+a)}}
\end{aligned}
$$

In order to find observational constraints on our model, we will study the particular case $n=2$ and $q=1$, which corresponds to the best choice of model in the power-law plateau inflation in Ref. [32]. In addition, we consider $\alpha$ and $V_{0}$, from the potential (1), and $C_{\phi}$, from the generalized inflaton decay ratio (2), as free parameters characterizing the model.

\subsection{Special case $n=2$ and $q=1$}

To compare the predictions of power-law plateau inflation in the cold and warm scenarios, we will restrict ourselves to the case $n=2$ and $q=1$, corresponding to the best choice of model in Ref. [32]. Moreover, for the generic parametrization of the inflaton decay rate (2), we consider the cases $a=3,1,0$, and -1 , which correspond to several dissipative ratios studied in the literature, but we consider $\alpha, V_{0}$ and $C_{\phi}$ to be free parameters. To put observational constraints on the parameters characterizing our model, we consider the essential condition for warm inflation, $T>H$, the condition for which the model evolves according to the strong regime, $R \gg 1$, and finally the two-dimensional marginalized joint confidence contours for $n_{\mathrm{S}}$ and $r$, at the 68 and 95\% CL and the amplitude of the scalar power spectrum by Planck 2015 data [21]. In addition, we will try to ascertain whether the predictions for the running of the scalar spectral index $\mathrm{d} n_{\mathrm{S}} / \mathrm{d} \ln k$ are consistent with the current bounds imposed by Planck.

\subsection{1 $a=3$}

In first place, for the special case $a=3$, i.e., for $\Gamma \propto T^{3} / \phi^{2}$, the scalar spectral index (35) becomes

$$
\begin{aligned}
n_{\mathrm{s}}= & 1+\frac{\left(3^{9} C_{\gamma}^{3} C_{\phi}^{-4}\right)^{\frac{1}{7}}}{7}\left(\frac{M_{\mathrm{p}}^{4}}{V_{0}}\right)^{\frac{2}{7}}\left(\frac{\phi}{M_{\mathrm{p}}}\right)^{-\frac{2}{7}} \\
& \times \frac{\left(4 \alpha^{2}+18\left(\frac{\phi}{M_{\mathrm{p}}}\right)^{2}\right)}{\left(\alpha^{2}+\left(\frac{\phi}{M_{\mathrm{p}}}\right)^{2}\right)} .
\end{aligned}
$$

From last expression we see that $n_{\mathrm{S}}$ is always greater than 1. Based on current observational data, for $\Lambda \mathrm{CDM}$ $+r+\mathrm{d} n_{\mathrm{S}} / \mathrm{d} \ln k$, the spectral index is measured to be $n_{\mathrm{s}}=$ $0.9667 \pm(68 \%$ CL, Planck TT + LowP). Hence, the inflaton decay ratio corresponding to $a=3$ is not suitable to describe a strong dissipative warm inflation scenario consistent with current observations. It is interesting that, for other inflaton potentials, the inflaton decay ratio $a=3$ describes a consistent warm inflationary dynamics (see Refs. [43,47,65-69]).

\section{$3.3 .2 a \neq 3$}

Figure 1 shows the ratio $\Gamma / 3 H$ and the tensor-to-scalar ratio $r$ as functions of the scalar spectral index $n_{\mathrm{s}}$ for the case $a=1$, i.e., $\Gamma(\phi, T)=C_{\phi} T$. To obtain the values to perform the plots, we have used three different values for $C_{\phi}$ parameter, fixed the values $C_{\gamma}=70$ and $M_{\mathrm{p}}=1$. For each value of $C_{\phi}$, we solve numerically the Eqs. (32) and (35) (after evaluating both equations at $\phi_{*}$ given by Eq. (31), which is a function of $V_{*}$ ) for $\alpha$ and $V_{0}$, considering the observational values $\mathcal{P}_{\mathcal{R}} \simeq$ $2 \times 10^{-9}, n_{\mathrm{s}} \simeq 0.9667$ [21] and fixing $V_{*}=10^{-12}$. In this way, for $C_{\phi}=4.39 \times 10^{-2}$, we obtain the values $\alpha=0.25$ and $V_{0}=1.03 \times 10^{-12}$, whereas for $C_{\phi}=9.45 \times 10^{-2}$, the solution is given by $\alpha=0.45$ and $V_{0}=1.09 \times 10^{-12}$. Finally, for $C_{\phi}=1.53 \times 10^{-1}$, we found that $\alpha=0.65$ and $V_{0}=1.19 \times 10^{-12}$. In this way, the $R\left(n_{\mathrm{S}}\right)$ and $r\left(n_{\mathrm{s}}\right)$ curves of Fig. 1 may be generated by plotting Eqs. (23), (35), and (39) parametrically (after being evaluated at $\phi_{*}$ given by Eq. (31)) with respect to $V_{*}$.

From the left panel, we observe that, for $C_{\phi} \geq 4.39 \times$ $10^{-2}$, the model evolves according to the strong regime, $R>1$. On the other hand, we find numerically that, for $C_{\phi} \geq 4.39 \times 10^{-2}$, the ratio $\frac{T}{H}$ becomes $\frac{T}{H} \gtrsim 79$ when $n_{\mathrm{s}} \simeq 0.9667$ (plot not shown). Hence, for $C_{\phi} \geq 4.39 \times 10^{-2}$ the essential condition for warm inflation, $\frac{T}{H}>1$, is always satisfied. Then the condition for which the model evolves in agreement with the strong regime gives us a lower limit on $C_{\phi}$. However, the essential condition for warm inflation does not impose any constraint on $C_{\phi}$. On the other hand, right panel of Fig. 1 shows the trajectories in the $n_{\mathrm{s}}-r$ plane along with the two-dimensional marginalized constraints at $68 \%$ and $95 \%$ C.L. on the parameters $r$ and $n_{\mathrm{s}}$, by Planck 2015 data [21]. Here, we observe that, for $C_{\phi} \geq 4.39 \times 10^{-2}$, the tensor-to-scalar ratio predicted by this model is always 


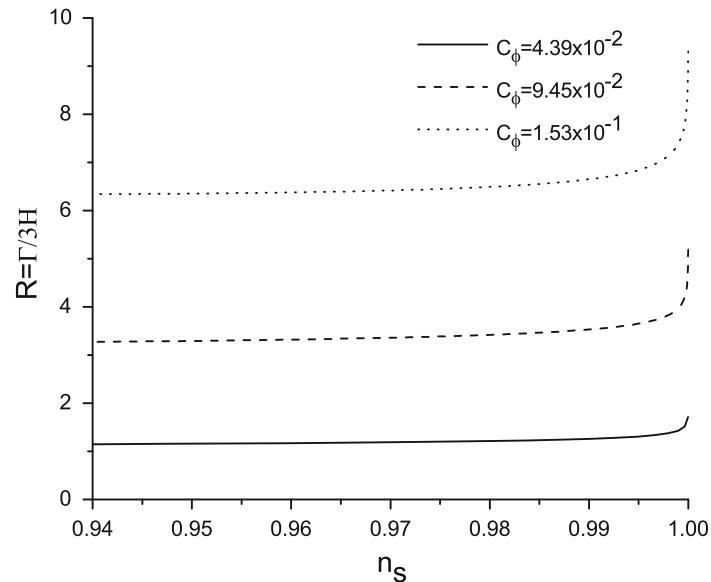

Fig. 1 Plots of $R=\Gamma / 3 H$ as a function of the scalar spectral index $n_{\mathrm{S}}$ (left) and the tensor-to-scalar $r$ as a function of the scalar spectral index $n_{\mathrm{s}}(r i g h t)$. For both plots, we have considered three different values of the parameter $C_{\phi}$ for the case $a=1$, i.e., $\Gamma \propto T$, assuming the model evolves according to the strong dissipative regime. In both pan-

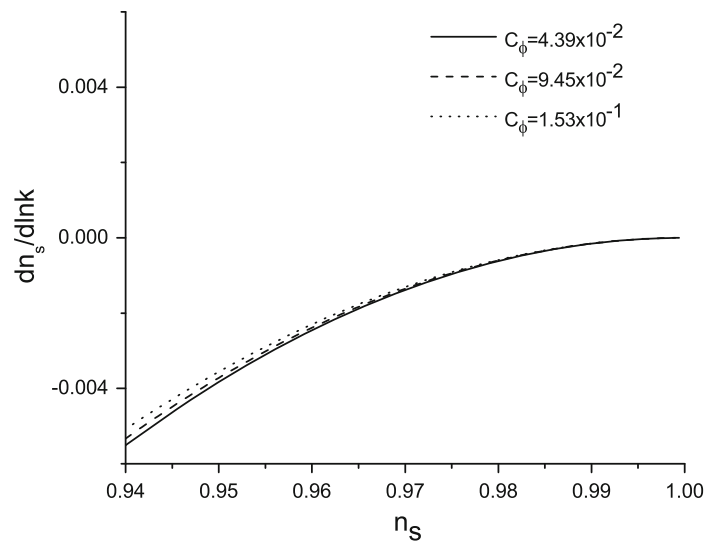

Fig. 2 Plots of the running of the scalar spectral index $\mathrm{d} n_{\mathrm{s}} / \mathrm{d} \ln k$ as a function of the scalar spectral index $n_{\mathrm{s}}$. For this plot, we have considered three different values of the parameter $C_{\phi}$ for the case $a=1$, i.e., $\Gamma \propto T$, assuming the model evolves according to the strong dissipative regime. The dotted, dashed, and solid lines correspond to the pairs of values $\left(\alpha=0.25, V_{0}=1.03 \times 10^{-12}\right),\left(\alpha=0.45, V_{0}=1.09 \times 10^{-12}\right)$, and $\left(\alpha=0.65, V_{0}=1.19 \times 10^{-12}\right)$, respectively. Additionally, we have fixed the values $C_{\gamma}=70$ and $M_{\mathrm{p}}=1$

consistent with the observational bound found by Planck, given by $r<0.168$ (95\% CL, Planck TT + LowP). In order to determine the prediction of this model regarding the running of the spectral index, Fig. 2 shows the trajectories in the $n_{\mathrm{s}}-\mathrm{d} n_{\mathrm{s}} / \mathrm{d} \ln k$ plane. Again we note that for $C_{\phi} \gtrsim 4.39 \times 10^{-2}$ the running of the spectral index predicted by the model is consistent with the bound found by Planck, given by $\mathrm{d} n_{\mathrm{s}} / \mathrm{d} \ln k=-0.0126_{-0.0087}^{+0.0098}(68 \% \mathrm{CL}$, Planck TT + LowP). After the previous analysis, we only were able to find a lower limit on $C_{\phi}$ as well as for $\alpha$ and $V_{0}$, given by $C_{\phi}=4.39 \times 10^{-2}, \alpha=0.25$ and $V_{0}=1.03 \times 10^{-12}$.

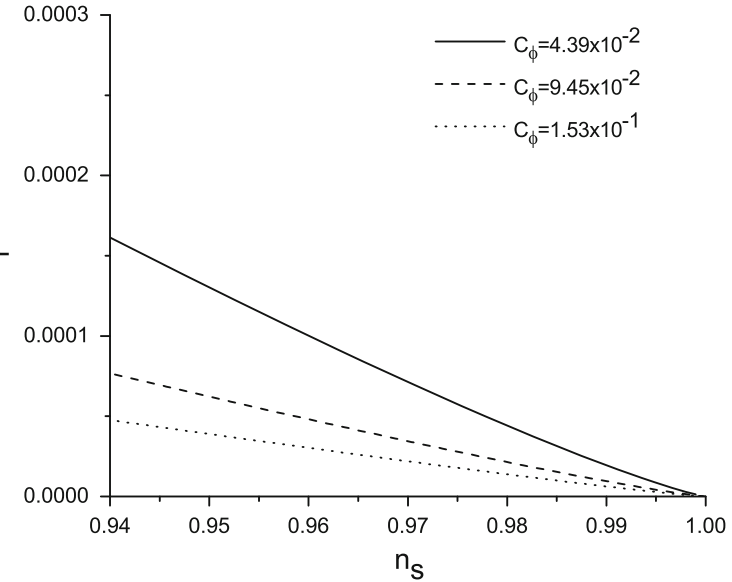

$e l s$, the dotted, dashed, and solid lines correspond to the pairs of values $\left(\alpha=0.25, V_{0}=1.03 \times 10^{-12}\right),\left(\alpha=0.45, V_{0}=1.09 \times 10^{-12}\right)$, and $\left(\alpha=0.65, V_{0}=1.19 \times 10^{-12}\right)$, respectively. In these plots, we have fixed the values $C_{\gamma}=70$ and $M_{\mathrm{p}}=1$

The same analysis can be done for the case $a=0$, for which $\Gamma \propto \phi$. Figure 3 shows the ratio $\Gamma / 3 H$ and the tensorto-scalar ratio $r$ as functions of the scalar spectral index. To obtain the values to perform the plots, we have used three different values for $C_{\phi}$ and followed the same procedure as the case $a=1$, solving numerically Eqs. (32) and (35) (after evaluating both equations at $\phi_{*}$ given by Eq. (31)) for $\alpha$ and $V_{0}$, considering $\mathcal{P}_{\mathcal{R}} \simeq 2 \times 10^{-9}, n_{\mathrm{s}} \simeq 0.9667$ [21], and fixing $V_{*}=10^{-12}$. In this way, for $C_{\phi}=7.49 \times 10^{-7}$, we obtain the values $\alpha=0.4$ and $V_{0}=1.02 \times 10^{-12}$, whereas for $C_{\phi}=9.67 \times 10^{-7}$, the solution is given by $\alpha=0.8$ and $V_{0}=1.06 \times 10^{-12}$. Finally, for $C_{\phi}=1.14 \times 10^{-6}$, we found that $\alpha=1.2$ and $V_{0}=1.11 \times 10^{-12}$. In this way, the $R\left(n_{\mathrm{s}}\right)$ and $r\left(n_{\mathrm{s}}\right)$ curves of Fig. 1 may be generated by plotting Eqs. (23), (35), and (39) parametrically with respect to $V_{*}$.

From the left panel of Fig. 3, the condition for the model evolves according to strong regime is satisfied for $C_{\phi} \geq 7.49 \times 10^{-7}$, which gives us a lower limit for $C_{\phi}$. Additionally, for $C_{\phi} \geq 7.49 \times 10^{-7}$ the condition for warm inflation, $\frac{T}{H}>1$, is always satisfied. In particular, for $C_{\phi}=7.49 \times 10^{-7}$, the ratio $\frac{T}{H}$ becomes $\frac{T}{H} \simeq 55$ when $n_{\mathrm{s}} \simeq 0.9667$. Then, just like the case $a=1$, for $a=0$ the essential condition for warm inflation does not impose any constraint on $C_{\phi}$. Moreover, from the right panel, for $C_{\phi} \geq 7.49 \times 10^{-7}$, the tensor-to-scalar ratio becomes $r \sim 10^{-5}$, but this value is still supported by the last data of Planck. For completeness, the running of the scalar spectral index becomes $\mathrm{d} n_{\mathrm{s}} / \mathrm{d} \ln k \simeq-0.001$ at $n_{\mathrm{s}} \simeq 0.9667$ (plot not shown). Then, for the case $a=1$, the previous analysis gives us only a lower limit for $C_{\phi}$ as well as for $\alpha$ and $V_{0}$, given by $C_{\phi}=7.49 \times 10^{-7}, \alpha=0.4$, and $V_{0}=1.02 \times 10^{-12}$, respectively. Despite this result, it is interesting to mention 


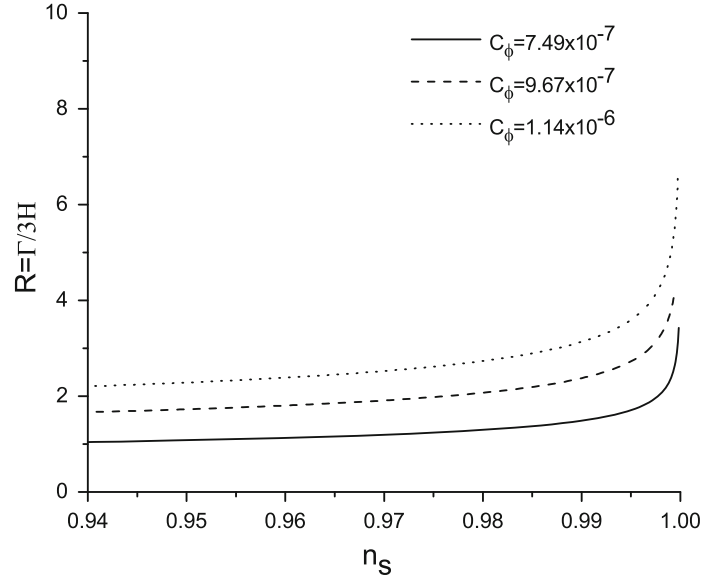

Fig. 3 Plots of $R=\Gamma / 3 H$ as a function of the scalar spectral index $n_{\mathrm{S}}$ (left) and the tensor-to-scalar $r$ as function of the scalar spectral index $n_{\mathrm{s}}(r i g h t)$. For both plots we have considered three different values of the parameter $C_{\phi}$ for the case $a=0$, i.e., $\Gamma \propto \phi$, assuming the model evolves according to the strong dissipative regime. In both pan-

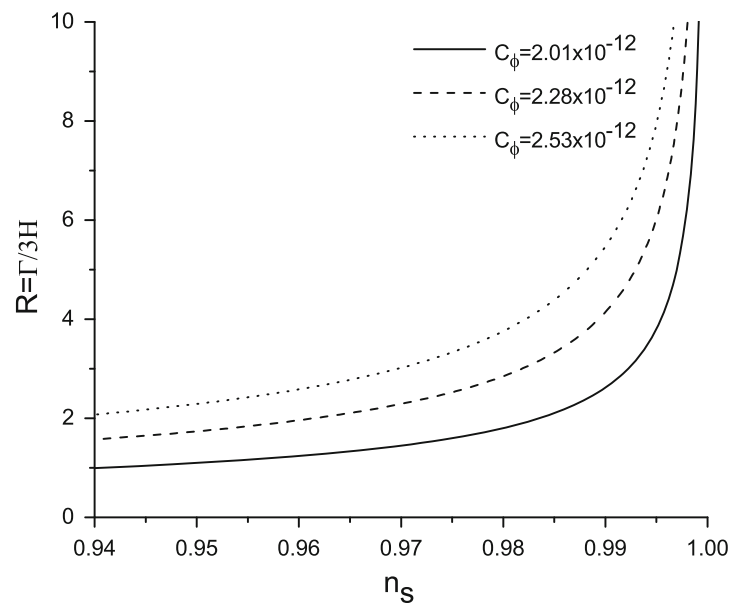

Fig. 4 Plots of the ratios $R=\Gamma / 3 H$ (left) and $T / H$ (right), both as functions of the scalar spectral index $n_{\mathrm{s}}$ (right). For both plots we have considered three different values of the parameter $C_{\phi}$ for the case $a=-1$, i.e., $\Gamma \propto \phi^{2} / T$, assuming the model evolves according to the strong dissipative regime. In both panels, the dotted, dashed, and solid

that, for this power-law plateau potential, the inflaton decay ratio $\Gamma \propto \phi$ describes a strong dissipative warm inflation scenario compatible with current observations. In previous works [65-69] it was found that the decay rate $\Gamma \propto \phi$ is not able to describe a consistent strong dissipate dynamics, since the predicted scalar spectral index is always greater than unity.

Following the same procedure as in the previous cases, for $a=-1$ we considered three different values for $C_{\phi}$. For $C_{\phi}=2.01 \times 10^{-12}$, we obtain the values $\alpha=1.5$ and $V_{0}=1.07 \times 10^{-12}$, whereas for $C_{\phi}=2.28 \times 10^{-12}$, the solution is given by $\alpha=1.95$ and $V_{0}=1.09 \times 10^{-12}$.

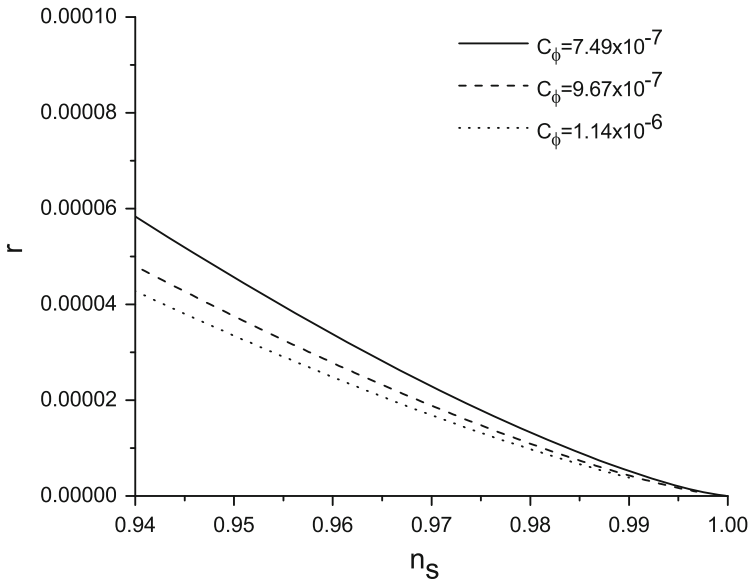

els, the dotted, dashed, and solid lines correspond to the pairs of values $\left(\alpha=0.4, V_{0}=1.02 \times 10^{-12}\right),\left(\alpha=0.8\right.$ and $\left.V_{0}=1.06 \times 10^{-12}\right)$, and $\left(\alpha=1.2, V_{0}=1.11 \times 10^{-12}\right)$, respectively. In these plots we have fixed the values $C_{\gamma}=70$ and $M_{\mathrm{p}}=1$

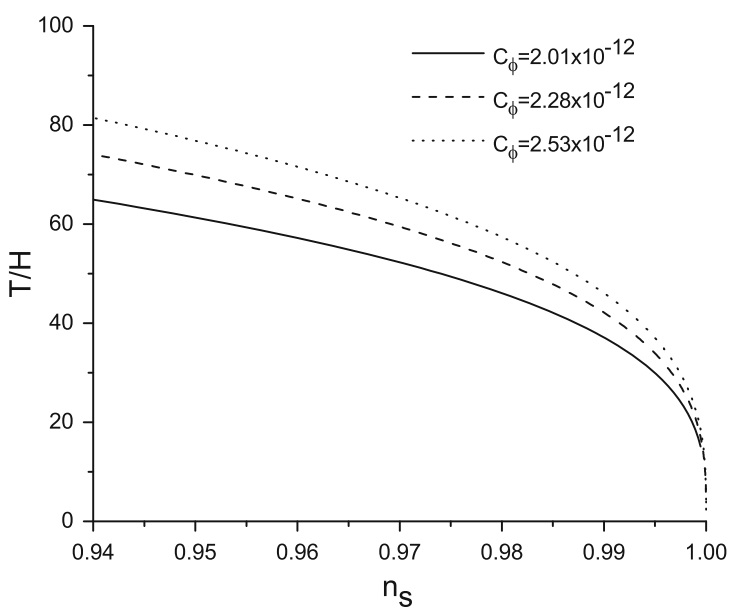

lines correspond to the pairs of values $\left(\alpha=1.5, V_{0}=1.07 \times 10^{-12}\right)$, $\left(\alpha=1.95\right.$ and $\left.V_{0}=1.09 \times 10^{-12}\right)$, and $\left(\alpha=2.4, V_{0}=1.13 \times 10^{-12}\right)$, respectively. In these plots we have fixed the values $C_{\gamma}=70$ and $M_{\mathrm{p}}=1$

Finally, for $C_{\phi}=2.53 \times 10^{-12}$, we found that $\alpha=2.4$ and $V_{0}=1.13 \times 10^{-12}$. Figure 4 shows the plots of the ratios $R=\Gamma / 3 H$ and $T / H$ as functions of the scalar spectral index $n_{\mathrm{s}}$. From the left panel, we see that for $C_{\phi} \geq 2.01 \times 10^{-12}$ the model takes place in the strong dissipative regime of warm inflation. Moreover, from the right panel, we observe that the essential condition for warm inflation $\frac{T}{H}>1$ is always guaranteed. In particular, for $C_{\phi}=2.01 \times 10^{-12}$, this ratio takes the value $\frac{T}{H} \simeq 60$ when $n_{\mathrm{s}} \simeq 0.9667$. Again, the $\frac{T}{H}$ plot does not impose any constraint on $C_{\phi}$. Regarding the predictions of this case in the $r-n_{\mathrm{S}}$ plane, for $C_{\phi}=2.01 \times 10^{-12}$, the tensor-to-scalar ratio becomes $r \sim 10^{-5}$, but this value is 
still supported by the last data of Planck by considering the two-dimensional marginalized joint confidence contours for $\left(n_{\mathrm{s}}, r\right)$, at the 68 and $95 \%$ C.L. (plot not shown). Finally, the predictions for the running of the spectral index are similar to previous ones, yielding $\mathrm{d} n_{\mathrm{s}} / \mathrm{d} \ln k \simeq-0.001$ at $n_{\mathrm{s}} \simeq 0.9667$ for all the values considered for $C_{\phi}$ (plot not shown). The result of this analysis yields only a lower limit for $C_{\phi}$ as well as for $\alpha$ and $V_{0}$, given by $C_{\phi}=2.01 \times 10^{-12}, \alpha=1.5$, and $V_{0}=1.07 \times 10^{-12}$, respectively. Just like the case $a=0$, the case $a=-1$ has an interesting feature, because yields a strong dissipative dynamics compatible with observations, since that in previous works [52,53,65-69], the inflaton decay rate $\Gamma \propto \frac{\phi^{2}}{T}$ is not able to describe a consistent strong dissipative dynamics.

\subsection{Discussion}

From the analysis carried out in Ref. [32], and provided that the model be distinguishable from monomial inflation, i.e., $\phi>\alpha M_{\mathrm{p}}$, the authors found that the best model choice in the power-law plateau inflation family correspond to the values $n=2$ and $q=1$. In a first approach and ensuring a sub-Planckian excursion for the inflaton through the potential, the maximum value allowed for $\alpha$ was found to be $\alpha=0.04$, and the values for the scalar spectral index and the tensor-to-scalar ratio at the Hubble-radius crossing correspond to $n_{\mathrm{s}}=0.97$ and $r=0.000157$. For our warm power-plateau model, in order to produce a strong dissipative dynamics, for each value of $a$, all the values obtained for $\alpha$ are greater than 0.04 , implying a trans-Planckian excursion of the inflaton field, but ensuring that $\phi>\alpha M_{\mathrm{p}}$. On the other hand, the predictions for the scalar spectral index are very similar for the cold and warm power-law plateau inflation models. Regarding the tensor-to-scalar ratio, in the warm inflation scenario, this quantity is suppressed by a factor $(T / H) R^{5 / 2}>1$ compared with standard cold inflation. In particular, for $a=1$, the tensor-to-scalar ratio is almost the same order compared to cold power-law plateau inflation. However, for $a=0$ and $a=-1$, the tensor-to-scalar ratio becomes smaller than for cold power-law plateau inflation.

In a second approach adopted in Ref. [32], the authors considered a trans-Planckian excursion of the inflaton field, obtaining values for $\alpha$ going from $\alpha=1$ up to $\alpha=5$, and the tensor-to-scalar ratio and the running of the scalar spectral index taking values from $r=0.004106$ up to $r=0.024412$, and from $\frac{\mathrm{d} n_{\mathrm{s}}}{\mathrm{d} \ln k}=-0.00057$ up to $\frac{\mathrm{d} n_{\mathrm{s}}}{\mathrm{d} \ln k}=-0.00051$, respectively. This implies that, for any value of $a$, the tensorto-scalar ratio for our warm power-law plateau inflation is always lower than the predicted by the cold scenario. On the other hand, the values predicted for the scalar spectral index in the cold and warm scenarios are very similar. In addition, it is interesting to mention that the running of the scalar spectral index $\frac{\mathrm{d} n_{\mathrm{s}}}{\mathrm{d} \ln k}$ predicted by our warm power-law plateau model is almost two orders of magnitude greater than the predicted by the cold scenario.

After the analysis performed previously for each value of $a$, we only found a lower limit on $C_{\phi}$ as well as for $\alpha$ and $V_{0}$, which means that we have a larger range of parameter values to enter in accordance with the Planck results and consistent with a strong dissipative dynamics. This degeneracy could be broken combining these results with the constraints on the inflationary observables related with non-Gaussianities, particularly the $f_{N L}$ parameter, since in warm inflation scenario these have different features when comparing with cold inflation [36]. Despite this issue, the predictions of warm powerlaw plateau inflation are comparable to those of power-law plateau cold inflation, however, the difference between both scenarios is a way to address the problem of reheating in cold power-law plateau inflation is provided by the warm inflation scenario.

\section{Conclusions}

In the present work, we have studied the consequences of considering a new family of single-field inflation models, called power-law plateau inflation, in the warm inflation scenario. As far we know, this is the first work in studying the dynamics of warm inflation by using the power-law plateau potential. In order to describe the dissipative effects during the inflationary expansion, we considered a generalized expression for the inflaton decay ratio given by $\Gamma(\phi, T)=C_{\phi} \frac{T^{a}}{\phi^{a-1}}$, where $a=3,1,0,-1$, denotes several inflaton decay ratios studied in the literature. We restricted ourselves only to the study of the strong dissipative regime, $R \gg 1$. For this dissipative regime, under the slow-roll approximation, we have studied the background as well as the perturbative dynamics. In particular, we have found the expressions for the scalar power spectrum, scalar spectral index and its running as well as the tensor-to-scalar ratio. Contrary to the standard cold inflation, in the warm inflation scenario it is not sufficient to consider only the constraints on the $r-n_{\mathrm{S}}$ plane, but we also have to consider the essential condition for warm inflation $T>H$ and the conditions for the model evolves under strong dissipative regime $R \gg 1$. For completeness, we study the predictions of our model regarding the running of the scalar spectral index, through the $n_{\mathrm{s}}-\mathrm{d} n_{\mathrm{s}} / \mathrm{d} \ln k$ plane.

To compare the predictions of power-law plateau inflation in the cold and warm scenarios, we restricted ourselves to the case $n=2$ and $q=1$, corresponding to the best choice of model in Ref. [32]. For this particular case, the inflaton decay $a=3$, i.e. $\Gamma \propto \frac{T^{3}}{\phi^{2}}$, fails in describe a strong dissipative 
dynamics consistent with current data, since the predicted value for the scalar spectral index is always greater than unity. We recall that, for the more representative potentials studied in the literature, the inflaton decay rate $a=3$ describes a warm inflationary dynamics consistent with current data. Regarding the predictions in the $n_{\mathrm{s}}-r$ and $n_{\mathrm{s}}-\mathrm{d} n_{\mathrm{s}} / \mathrm{d} \ln k$ planes, for $a=1$, the tensor-to-scalar ratio and the running of the spectral index becomes $r \simeq 10^{-4}$ and $\frac{\mathrm{d} n_{\mathrm{s}}}{\mathrm{d} \ln k} \simeq 0.002$, respectively, whereas for both the cases $a=0$ and $a=$ -1 , these inflationary observables become $r \simeq 10^{-5}$ and $\frac{\mathrm{d} n_{\mathrm{s}}}{\mathrm{d} \ln k} \simeq 0.001$, being consistent with current bounds imposed by Planck for $\Lambda \mathrm{CDM}+r+\mathrm{d} n_{\mathrm{s}} / \mathrm{d} \ln k$. It is interesting to mention that, for other kinds of potentials already studied in the warm inflaton scenarios, the decay ratios $a=0$ and $a=-1$ predicted a scalar spectral index always greater than unity. On the other hand, for any value of $m$, the condition for the model evolves according to the strong dissipative regime sets the lower limit for the disipative parameter $C_{\phi}$ as well for $\alpha$ and $V_{0}$. However, the essential condition for warm inflation to occur, $T>H$ neither the Planck data, by considering the two-dimensional marginalized constraints at $68 \%$ and 95\% C.L. on the parameters $r$ and $n_{\mathrm{s}}$, do not impose any constraints on the model for this dissipative regime, obtaining a lower limit on $C_{\phi}$ as well as for $\alpha$ and $V_{0}$. However, if we consider the observational constraints on the inflationary observables related with non-Gaussianities, particularly the $f_{N L}$ parameter, this degenerancy in the parameters could be broken.

Comparing our warm power-law plateau inflation model with the standard one, we found that the strong dissipative warm inflation dynamics is only consistent with a transPlanckian incursion of the inflaton potential, according with second approach adopted in [32], ensuring that this powerlaw plateau potential be distinguishable from monomial inflation. For this trans-Planckian evolution of the inflaton, and for any value of $a$, the tensor-to-scalar ratio for our warm power-law plateau inflation is always lower than predicted by the cold scenario. On the other hand, the values predicted for the scalar spectral index in the cold and warm scenarios become similar, however, the running of the scalar spectral index $\frac{\mathrm{d} n_{\mathrm{s}}}{\mathrm{d} \ln k}$ is almost two orders of magnitude greater than predicted by the cold scenario. We have shown that warm power-law plateau inflaton, with decay ratios parametrized by $a=1,0$, and -1 , is consistent with a strong dissipative dynamics and predicts values for the scalar spectral index, the running of the scalar spectral index, and the tensor-to-scalar ratio consistent with current bounds imposed by Planck, for $\Lambda \mathrm{CDM}+r+\mathrm{d} n_{\mathrm{s}} / \mathrm{d} \ln k$.

Acknowledgements N.V. was supported by Comisión Nacional de Ciencias y Tecnología of Chile through FONDECYT Grant No. 3150490. Finally, we wish to thank the anonymous referee for her/his valuable comments, which have helped us to improve the presentation in our manuscript.
Open Access This article is distributed under the terms of the Creative Commons Attribution 4.0 International License (http://creativecomm ons.org/licenses/by/4.0/), which permits unrestricted use, distribution, and reproduction in any medium, provided you give appropriate credit to the original author(s) and the source, provide a link to the Creative Commons license, and indicate if changes were made.

Funded by $\mathrm{SCOAP}^{3}$.

\section{References}

1. A.A. Starobinsky, Phys. Lett. 91B, 99 (1980)

2. A. Guth, Phys. Rev. D 23, 347 (1981)

3. K. Sato, Mon. Not. R. Astron. Soc. 195, 467 (1981)

4. A.D. Linde, Phys. Lett. B 108, 389 (1982)

5. A.D. Linde, Phys. Lett. B 129, 177 (1983)

6. A. Albrecht, P.J. Steinhardt, Phys. Rev. Lett. 48, 1220 (1982)

7. A.D. Linde, Phys. Lett. B 129, 177 (1983)

8. A.A. Starobinsky, JETP Lett. 30, 682 (1979)

9. V.F. Mukhanov, G.V. Chibisov, JETP Lett. 33, 532 (1981)

10. S.W. Hawking, Phys. Lett. B 115, 295 (1982)

11. A. Guth, S.-Y. Pi, Phys. Rev. Lett. 49, 1110 (1982)

12. A.A. Starobinsky, Phys. Lett. B 117, 175 (1982)

13. J.M. Bardeen, P.J. Steinhardt, M.S. Turner, Phys. Rev. D 28, 679 (1983)

14. D. Larson et al., Astrophys. J. Suppl. 192, 16 (2011)

15. C.L. Bennett et al., Astrophys. J. Suppl. 192, 17 (2011)

16. N. Jarosik et al., Astrophys. J. Suppl. 192, 14 (2011)

17. G. Hinshaw et al. [WMAP Collaboration], Astrophys. J. Suppl. 208, 19 (2013)

18. P.A.R. Ade et al. [Planck Collaboration], Astron. Astrophys. 571, A16 (2014)

19. P.A.R. Ade et al. [Planck Collaboration], Astron. Astrophys. 571, A22 (2014)

20. P.A.R. Ade et al. [Planck Collaboration], Astron. Astrophys. 594, A13 (2016)

21. P.A.R. Ade et al. [Planck Collaboration], Astron. Astrophys. 594, A20 (2016)

22. L. Kofman, A.D. Linde, A.A. Starobinsky, Phys. Rev. Lett. 73, 3195 (1994)

23. L. Kofman, A.D. Linde, A.A. Starobinsky, Phys. Rev. D 56, 3258 (1997)

24. R. Allahverdi, R. Brandenberger, F.Y. Cyr-Racine, A. Mazumdar, Ann. Rev. Nucl. Part. Sci. 60, 27 (2010)

25. M.A. Amin, M.P. Hertzberg, D.I. Kaiser, J. Karouby, Int. J. Mod. Phys. D 24, 1530003 (2014)

26. J. Martin, C. Ringeval, R. Trotta, V. Vennin, JCAP 1403, 039 (2014)

27. F. Bezrukov, A. Magnin, M. Shaposhnikov, S. Sibiryakov, JHEP 1101, 016 (2011)

28. R. Kallosh, A. Linde, D. Roest, JHEP 1311, 198 (2013)

29. R. Kallosh, A. Linde, D. Roest, JHEP 1408, 052 (2014)

30. K. Dimopoulos, Phys. Lett. B 735, 75 (2014)

31. K. Dimopoulos, PoS PLANCK 2015, 037 (2015)

32. K. Dimopoulos, C. Owen, Phys. Rev. D 94(6), 063518 (2016)

33. I.G. Moss, Phys. Lett. B 154, 120 (1985)

34. A. Berera. Phys. Rev. Lett. 75, 3218 (1995)

35. A. Berera, Phys. Rev. D 55, 3346 (1997)

36. M. Bastero-Gil, A. Berera, I.G. Moss, R.O. Ramos, JCAP 1412(12), 008 (2014)

37. M. Bastero-Gil, A. Berera, N. Kronberg, JCAP 1512(12), 046 (2015)

38. G. Panotopoulos, N. Videla, Eur. Phys. J. C 75(11), 525 (2015)

39. M. Bastero-Gil, A. Berera, R.O. Ramos, J.G. Rosa, Phys. Rev. Lett. 117(15), 151301 (2016)

40. L. Visinelli, JCAP 1607(07), 054 (2016) 
41. Y. Gim, W. Kim, JCAP 1611(11), 022 (2016)

42. G. Øyvind, Universe 2(3), 20 (2016)

43. M. Benetti, R.O. Ramos, arXiv:1610.08758 [astro-ph.CO]

44. Z.P. Peng, J.N. Yu, X.M. Zhang, J.Y. Zhu, Phys. Rev. D 94(10), $103531(2016)$

45. K. Sayar, A. Mohammadi, L. Akhtari, K. Saaidi, Phys. Rev. D 95(2), 023501 (2017)

46. I.G. Moss, C. Xiong, arXiv:hep-ph/0603266

47. M. Bastero-Gil, A. Berera, R.O. Ramos, J.G. Rosa, JCAP 1301, 016 (2013)

48. S. Bartrum, M. Bastero-Gil, A. Berera, R. Cerezo, R.O. Ramos, J.G. Rosa, Phys. Lett. B 732, 116 (2014)

49. A. Berera, I.G. Moss, R.O. Ramos, Rep. Prog. Phys. 72, 026901 (2009)

50. M. Bastero-Gil, A. Berera, Int. J. Mod. Phys. A 24, 2207 (2009)

51. A. Berera, M. Gleiser, R.O. Ramos, Phys. Rev. Lett. 83, 264 (1999)

52. A. Berera, M. Gleiser, R.O. Ramos, Phys. Rev. D 58, 123508 (1998)

53. J. Yokoyama, A. Linde, Phys. Rev D 60, 083509 (1999)

54. Y. Zhang, JCAP 0903, 023 (2009)
55. M. Bastero-Gil, A. Berera, R.O. Ramos, JCAP 1109, 033 (2011)

56. I.G. Moss, Phys. Lett. B 154, 120 (1985)

57. A. Berera, Phys. Rev. D 54, 2519 (1996)

58. A. Berera, L.Z. Fang, Phys. Rev. Lett. 74, 1912 (1995)

59. A. Berera, Nucl. Phys. B 585, 666 (2000)

60. L.M.H. Hall, I.G. Moss, A. Berera, Phys. Rev. D 69, 083525 (2004)

61. I.G. Moss, C. Xiong, JCAP 0811, 023 (2008)

62. R.O. Ramos, L.A. da Silva, JCAP 1303, 032 (2013)

63. R.O. Ramos, Astrophys. Space Sci. Proc. 45, 283 (2016)

64. G.B. Arfken, H.J. Weber, F.E. Harris, Mathematical methods for physicists: a comprehensive guide (Academic Press/Elsevier, Waltham, MA, 2013)

65. R. Herrera, M. Olivares, N. Videla, Phys. Rev. D 88, 063535 (2013)

66. R. Herrera, M. Olivares, N. Videla, Int. J. Mod. Phys. D 23(10), 1450080 (2014)

67. R. Herrera, N. Videla, M. Olivares, Phys. Rev. D 90(10), 103502 (2014)

68. R. Herrera, N. Videla, M. Olivares, Eur. Phys. J. C 75(5), 205 (2015)

69. R. Herrera, N. Videla, M. Olivares, Eur. Phys. J. C 76(1), 35 (2016) 